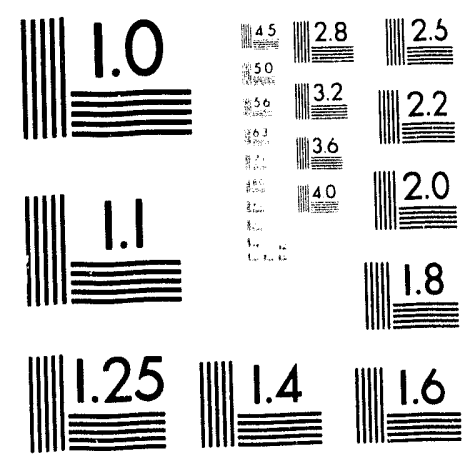



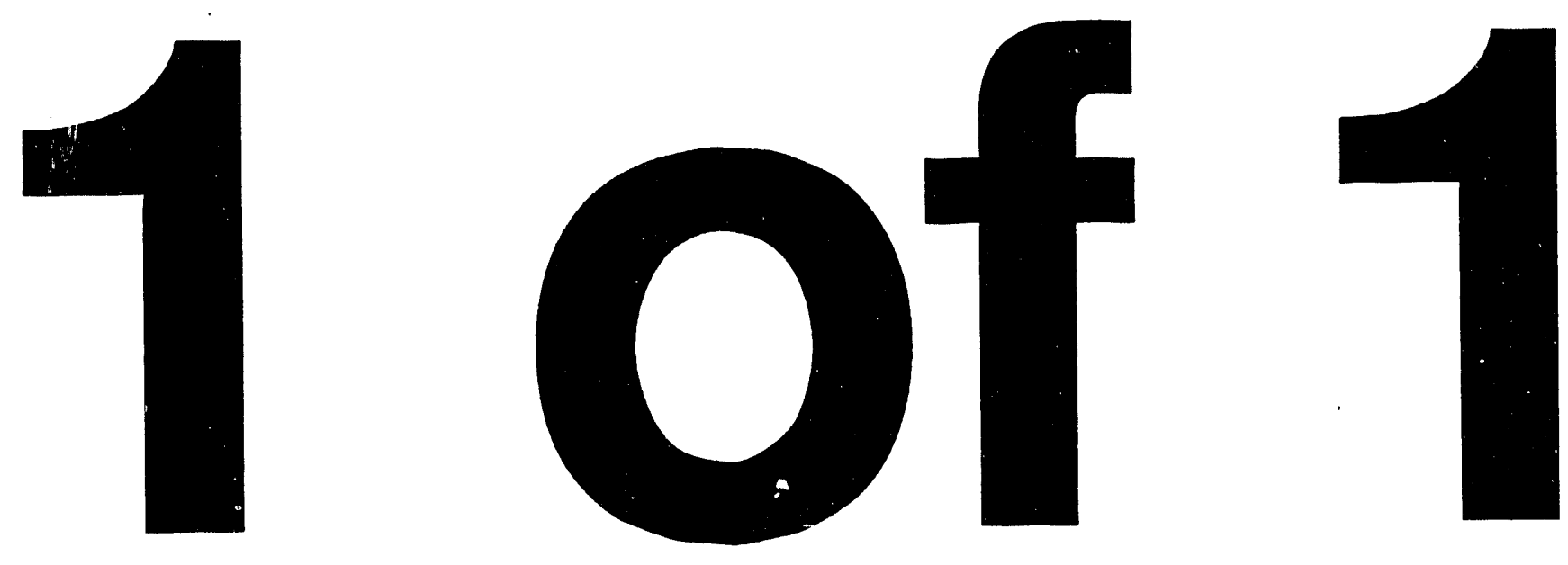
UCRL-JC-113400

PREPRINT

\section{Computer Simulation of Molecular Response at a Shock Front}

James Belak

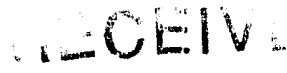

OCT 141993

OSTI

This paper was prepared for submittal to the Proceedings of the Joint AIRAPT/APS Conference

Colorado Springs, $C O$

June 28-July 2, 1993

July 1993

This is a preprint of a paper intended for publication in a journal or proceedings. Since changes may be made before publication, this preprint is made available with the understanding that it will not be cited or reproduced without the permission of the author. 


\section{DISCLAIMER}

This document was prepared as an account of work sponsored by an agency of the United States Government. Neither the United States Government nor the University of California nor any of their employees, makes any warranty, express or implied, or assumes any legal liability or responsibility for the accu racy, completeness, or usefulness of any information, apparat us, product, or process disclosed, or represents that its use would not infringe privately owned rights. Reference herein to any specific commercial products, process, or service by trade name, trademark, manufacturer, or otherwise, does not necessarily constitute or imply its endorsement, recommendation, or favoring by the United States Government or the University of California. The views and opinions of authors expressed herein do not necessarily state or reflect those of the United States Government or the University of Califomia, and shall not be used for advertising or product endorsement purposes. 


\title{
COMPUTER SIMULATION OF MOLECULAR RESPONSE AT A SHOCK FRONT
}

\author{
J. Belak \\ Lawrence Livermore National Laboratory \\ Livermore, California 94551
}

Molecular dynamics simulations of the response of small molecules at a shock front are presented. The simulations are performed in the reference frame of the shock and the results provide a unique insight into the sequence of states through which the material transforms during shock loading. The calculated up-pumping time (time for the translational and vibrational kinetic energy to equilibrate) for small molecules is quite fast, a few picoseconds for butane and about 10 ps for nitromethane. This is somewhat faster than the 100 ps suggested by recent experiment.

\section{INTRODUCTION}

The purpose of this paper is to address one of the outstanding questions in shock physics; that is, what is the sequence of states through which the material transforms in going from the initial state in front of the shock to the final high-temperature and high-pressure state behind the shock? For example, during shock loading of high explosive molecules, such as $H M X$, the shock wave dumps a huge initiation energy into the translational motion of the

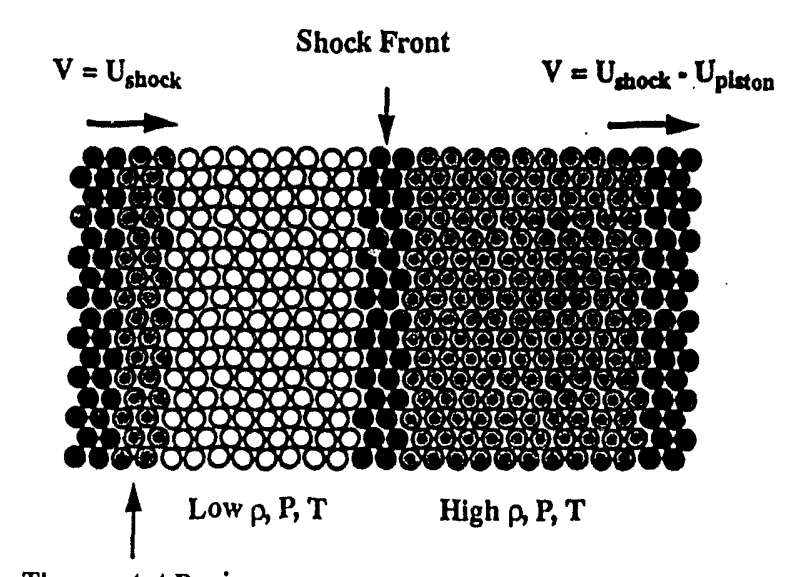

Thermostat Region

Fig 1. A schematic illustration of the constant velocity boundary condition used to simulate shock propagation within the reference frame of the shock. molecules. This energy is slowly transferred into internal vibrational motion of the molecules, weakening the chemical bonds. This process, known as multiphonon up-pumping, continues to be a hot research topic. ${ }^{1}$

In this work we present molecular dynamics simulations of the response of small molecules at a shock front. The simulations are performed in the reference frame of the shock and the results suggest that the up-pumping time (time for the translational and vibrational kinetic energy to equilibrate) for small molecules is quite fast, a few picoseconds for butane and about $10 \mathrm{ps}$ for nitromethane. This is somewhat faster than the $100 \mathrm{ps}$ suggested by recent experiment. ${ }^{1}$

\section{MODEL}

The molecular dynamics (MD) computer simulation method ${ }^{2}$ is an ideal approach to study this and related problems such as the reactive dynamics of chemically-sustained shocks. ${ }^{3}$ The MD method has been applied to the study of shock problems with significant success. ${ }^{4} 10$ In these previous studies the shock was generated by either driving a fixed wall into the material (material reference frame) or

\section{MASTER}

DISTRBSUTON OF THIS DOCUMENT IS UNLMTIED 
driving the material into a stationary wall (piston reference frame). In this work we take an alternate approach and perform the simulation within the reference frame of the shock. This coordinate frame is especially useful. For in this frame only, the flux of mass, momentum and energy have constant values everywhere when the profile is steady. ${ }^{11}$

We perform the simulation as follows: ${ }^{12}$ all atoms within a boundary region (see Figure 1) are constrained to propagate at a constant velocity. Atoms are continually inserted from the left, while atoms that leave the box are discarded-the system is open. The system is initially equilibrated at the desired temperature and density using the standard periodic

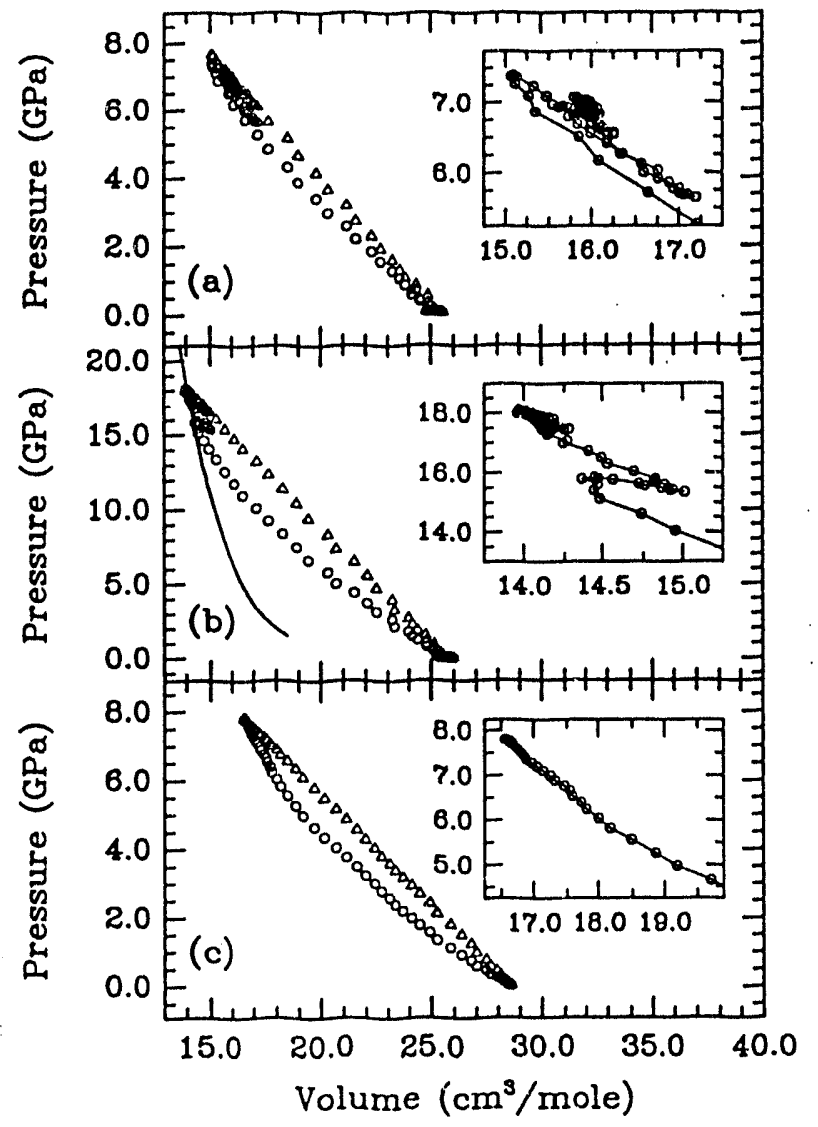

Fig 2. The sequence of states in P-V space that simulated argon follows as it goes through a shock front. In each case, the inset shows the approach to the final state. boundary conditions. The final configuration from this periodic simulation is used to generate the configuration of incoming material. In addition to sampling the incoming velocities from a Maxwell-Boltzmann distribution, we pass this incoming material through a small thermostat region to assure that the initial temperature is as desired. The remaining interior atoms are purely Newtonian and we retain the periodic boundary condition within the plane of the shock. The shock wave simulation is performed by imposing a constant velocity $\left(U_{\text {shock }}\right)$ to the incoming material and a slower constant velocity $\left(U_{\text {shock }}-U_{\text {piston }}\right)$ to the departing material. As we increase the piston velocity, material piles up at the departing boundary until, above a critical velocity, a sharp density discontinuity propagates into the simulation cell (to the left in Figure 1). We associate this density discontinuity with a shock front. By decreasing the piston velocity, we make the shock front stationary with respect to the simulation cell and, in doing so, we are able to study shock propagation for arbitrarily long periods of time.

\section{RESULTS AND DISCUSSION}

To facilitate comparison with experiment, we have introduced cross-sectional bins (1000 per simulation cell) into which to measure physical observables. The averages presented here are relative (in position) to the shock front. One observable of primary interest is the average flow velocity. We find the velocity to maintain a well-defined plateau ( $\left.U_{\text {shock }}\right)$ in front of the shock, decreasing rapidly through the shock front, and maintaining a welldefined plateau $\left(U_{\text {shock }}-U_{\text {piston }}\right)$ well behind the shock.

In Figure 2, we show the path in P-V space for three simulations of solid and liquid argon using the potential model due to Ross et al, ${ }^{13}$ which gives excellent agreement with the experimental Hugoniot up to shock velocities 
of $9 \mathrm{~km} / \mathrm{s}$. The open triangles represent the longitudinal component of the pressure tensor $\left(P_{x x}\right)$ which varies linearly with volume through the shock front. This is a direct consequence of conservation of momentum. The straight line is known as the Raleigh line and the area beneath this line is equal to the change in internal energy between the initial and final states. ${ }^{11}$ The pressure, however, includes both the longitudinal and transverse components of the pressure tensor $\left(P=\left(P_{x x}{ }^{+-}\right.\right.$ $\left.P_{y y}+P_{z z}\right) / 3$ ) and the sequence of pressure states (open circles) does not vary linearly with volume.

The sequence of pressure states for a $7 \mathrm{GPa}$ shock in solid argon is shown in Figure 2a. At the shock front, the pressure over-shoots the final state, then oscillates with diminishing amplitude, finally rising slightly at nearly constant volume as the system melts. In Figure $2 b$, we show the sequence of states for a stronger shock (18 GPa) in the solid. The pressure, while increasing in the solid, does not overshoot the final state and the melting does not occur at constant volume. We also show in this figure, for comparison, our calculated Hugoniot curve (locus of final PV states) for shocks along the [100] axis in solid argon. Finally, we show in Figure 2c, the sequence of states for a

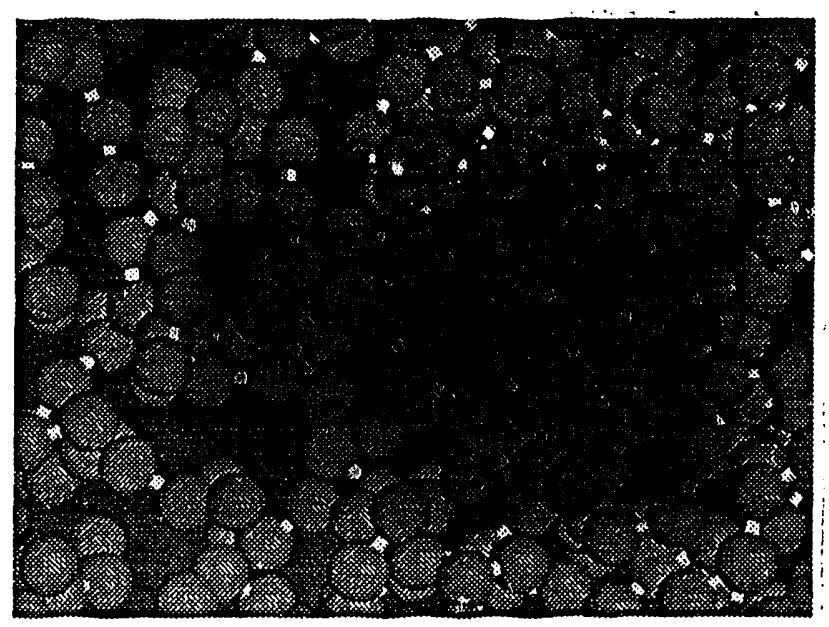

Fig 3. A 10 GPa shock wave in liquid butane.

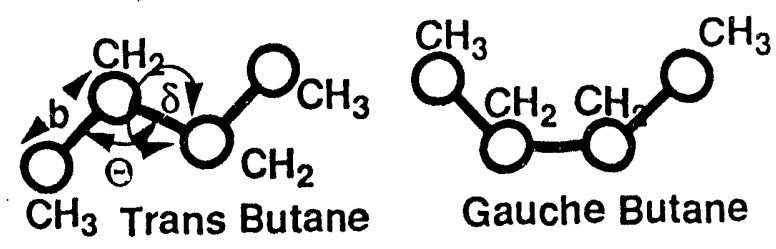

Fig 4. The trans and gauche configurations of butane.

liquid-state shock of similar strength to the solid-state shock shown in Figure 2a. The pressure rises smoothly with decreasing volume, though we note a slight kink in the curve near the final state, possibly due to the fluid responding in a solid-like manner during the short time scale involved in loading and later relaxing out of the non-equilibrium state.

In Figure 3, we show a $0.6 \mathrm{~nm}$ thick crosssectional snapshot of a $10 \mathrm{GPa}$ shock wave in liquid butane. Butane is the simplest molecule exhibiting all of the degrees of freedom (bond length, angles and torsions) required to simulate larger high-explosive molecules. The thermodynamics and potential models are wellknown. ${ }^{14}$ Here, we use a united-atom approximation. In the room temperature liquid in front of the shock, butane forms a trans config-

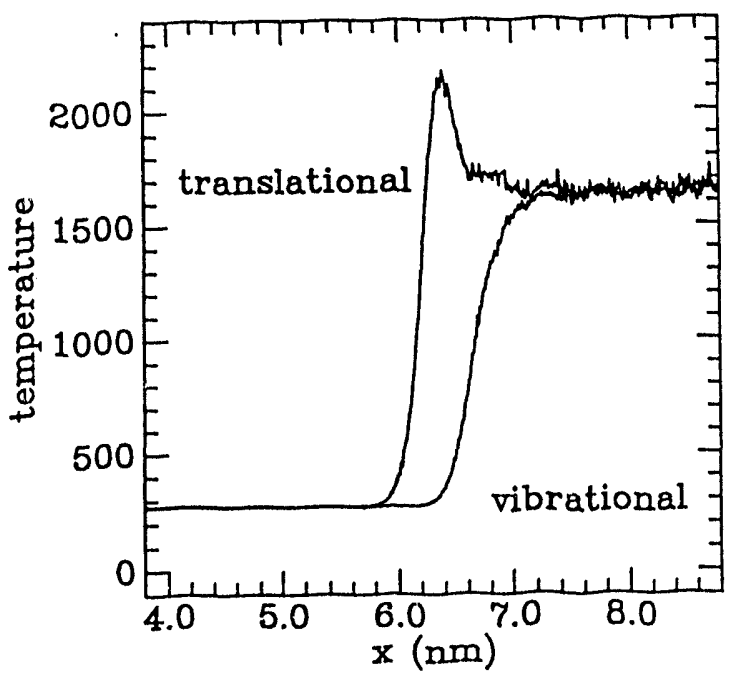

Fig 5. The translational and vibrational kinetic energy of liquid butane as a function of position through a $10 \mathrm{GPa}$ shock. 
uration as shown in Figure 4, while behind the shock we find a significant number of gauche defects. Of greater interest is the distribution of kinetic energy into translational and vibrational degrees of freedom. This is shown in Figure 5. For each bin we calculate the expectation value of the center of mass (CM) kinetic energy, rotational kinetic energy about the $\mathrm{CM}$, and the total kinetic energy. In Figure 5 the curve marked translational is the sum of the $\mathrm{CM}$ and rotational kinetic energy and the curve marked vibrational is the difference between the translational energy and the total kinetic energy. The translational energy leads the vibrational energy by a few nanometers (a few picoseconds). Preliminary simulations using available intermolecular ${ }^{15}$ and intramolecular ${ }^{16}$ potential models for nitromethane suggest a lead time of nearly 10 ps.

In summary we note that our simulations give unique insight into the sequence of states through which the material transforms during shock loading. The calculated up-pumping time for small molecules is quite fast, a few picoseconds for butane and nearly 10 ps for nitromethane. This is somewhat faster than suggested by recent experiment. In future work we plan to project the molecular motion onto the normal coordinates for vibrational motion. This should lead to further insight into the doorways for energy transport into the molecule.

\section{ACKNOWLEDGEMENTS}

The author is indebted to Dave Boercker, Marvin Ross and Clark Souers for many useful suggestions and enlightening discussions. This work was performed at Lawrence Livermore National Laboratory under the auspices of the U.S. DOE through Contract No. W-7405ENG-48.

\section{REFERENCES}

[1] A. Tokmakoff, M.D. Fayer, and D.D. Dlott, J.
Phys. Chem. 97, 1901 (1993); and references therein.

[2] M.P. Allen and D.J. Tildesley, Computer Simulation of Liquids, (Clarendon Press, Oxford, 1987).

[3] D.H. Robertson, D.W. Brenner, and C.T. White, Phys. Rev. Lett. 67, 3132 (1991).

[4] D.H. Tsai and C.W. Beckett, J. Geophys. Res. 71, 2601 (1966).

[5] D.H. Tsai and R.A. MacDonald, F. Phys. C 11, L365 (1978).

[6] A. Paskin and G.J. Dienes, J. Appl. Phys. 43, 1605 (1972).

[7] A. Paskin, A. Gohar, and G.J. Dienes, J. Phys. C 11, L857 (1978).

[8] B.L. Holian and G.K. Straub, Phys. Rev. Lett. 43, 1598 (1979).

[9] B.L. Holian, W.G. Hoover, B. Moran, and G.K. Straub, Phys. Rev. A 22, 2798 (1980).

[10] B.L. Holian, Phys. Rev. A 37, 2562 (1988).

[11] Ya. B. Z.jl'dovich and Yu. P. Razier, Physics of Shock Waves and High-Temperature Hydrodynamic Phenomena (Academic Press, New York, 1966).

[12] J. Belak, Conservation of Momentum through a Shock Front, Lawrence Livermore National Laboratory Report UCRL-JC-109989, (1992).

[13] M. Ross, H.K. Mao, P.M. Bell, and J.A. Xu, J. Chem. Phys. 85, 1028 (1986).

[14] J.-P. Ryckaert and A. Bellemans, Chem. Phys. Lett. 30, 123 (1975).

[15] J. Caillet and P. Claverie, Acta Cryst. A31, 448 (1975).

[16] B.M. Rice and D.L. Thompson, J. Chem. Phys. 93, 7986 (1990). 

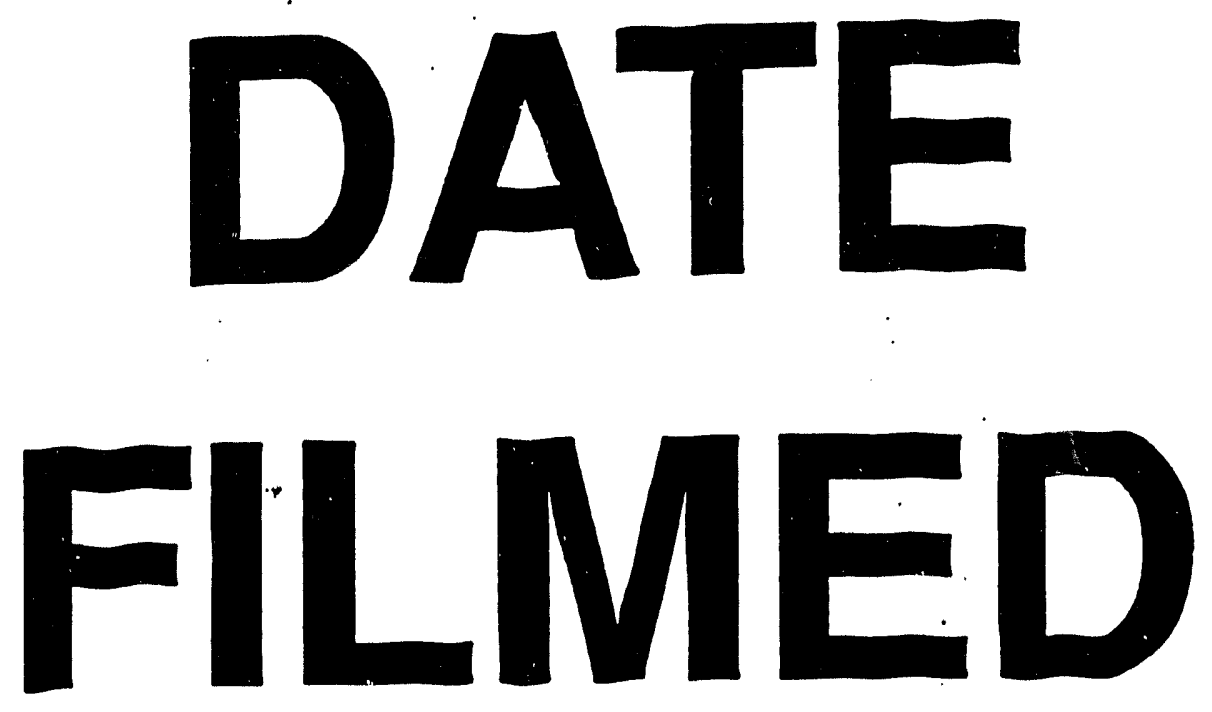

$12 / 8 / 93$
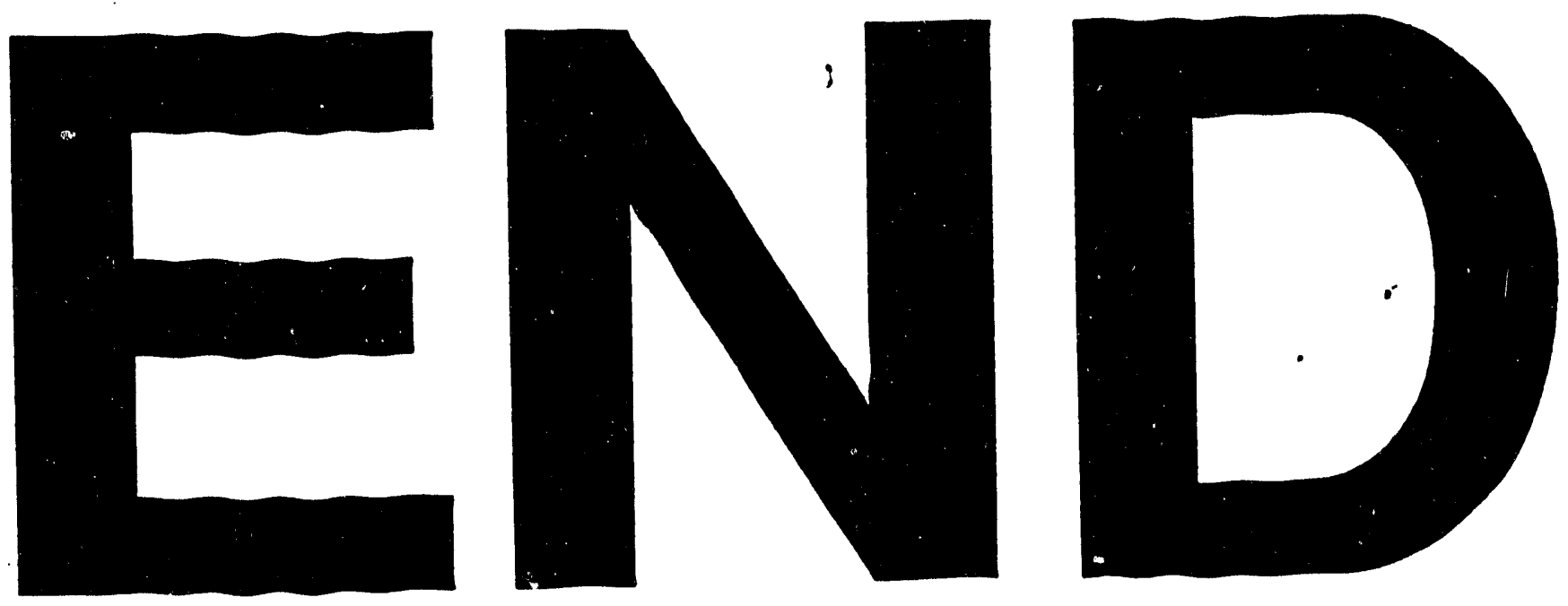


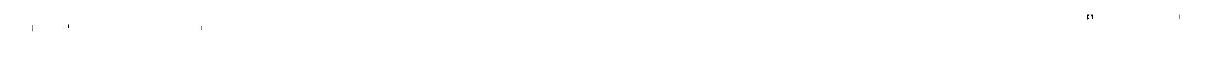

$+2+1$ 